\title{
An Analysis of Plug and Play Business Software
}

\author{
Andreas Jacobsson and Paul Davidsson \\ Department of Systems and Software Engineering, School of Engineering, \\ Blekinge Institute of Technology, SE-372 25 Ronneby, Sweden \\ \{andreas.jacobsson, paul.davidsson\}@bth.se
}

\begin{abstract}
The ability to realize the potential of innovations is important for societies to be successful. The core of the concept of Plug and Play Business is an integrated set of ICT-tools that support innovators in turning their ideas into businesses by forming virtual enterprises for inter-organizational and interoperable collaboration. In this paper, we analyze the concept of Plug and Play Business and present a formalization of relevant aspects, such as the different tasks involved in virtual enterprise formation and collaboration. We explore the functional requirements of Plug and Play Business software, and make an assessment of the usefulness of existing technologies for its implementation.
\end{abstract}

\section{Introduction}

The fact that innovations are important to create both private and social values, including economic growth and employment is well-known. From an innovator's perspective there are some common obstacles for realizing the potential of innovations, such as shortage of time to spend on commercialization activities, lack of business knowledge, underdeveloped business network, and limited financial resources [18]. Thus, the innovator requires support to develop the innovation into business, something often seen as the specific role of the entrepreneur, which is to search, discover, evaluate opportunities and marshal the financial resources necessary, among other things [13].

The concept of Plug and Play Business [9] relies on an integrated set of ICTtools that supports the creation and management of businesses despite the obstacles mentioned above. We envision Plug and Play Business as a helpful ICT-measure to enterprises, small and medium-sized enterprises (SME) in particular, in realizing innovations. ICT-infrastructures for business creation and collaboration are particularly relevant to SMEs, due to their size and high specialization and 
flexibility. While allowing themselves to maintain their business independence, SMEs are able to reach otherwise unreachable markets and to take advantage of economies of scale with the support of ICT [5]. After having deployed the Plug and Play Business software, companies are connected to a networked community where all participants share one common goal; namely to increase business. In that way, the purpose of Plug and Play Business is to stimulate the realization of innovations without interfering with the individual goals of the Plug and Play Business companies. Together with the autonomy, heterogeneity, and possibly conflicting goals of the involved parties of a Plug and Play Business community, this requires ICT-solutions that are able to handle dynamically evolving and distributed business partnerships and processes that cross the borders of various enterprises. Thus, the interoperability between the information systems of the involved enterprises belongs to the technological core of the concept of Plug and Play Business.

This paper partially builds on previous work [10] in which we have made a proof of concept with regards to interorganizational interoperability of enterprise systems. In Carlsson et al. [6], we analyzed the security risks related to such collaboration. In this paper, we first describe the concept of Plug and Play Business and identify the requirements of the supporting software. Then we provide a formal model of Plug and Play Business, including crucial aspects such as virtual enterprise formation and collaboration. The lack of appropriate theoretic definitions and formal models has been argued to be one of the main weaknesses in the area of virtual enterprises [2,3]. We also review current state of the art and identify useful technologies for the implementation of Plug and Play Business software. We conclude with some suggestions for future work. However, we begin by describing the concept of Plug and Play Business, its functional requirements, and by exploring its quality attributes.

\section{The Concept and Requirements of Plug and Play Business}

Central to Plug and Play Business is the concept of virtual enterprises (VE), or more generally collaborative networks [2]. A VE is a temporary alliance of enterprises that come together to share skills or core competencies and resources in order to better respond to business opportunities, and whose cooperation is supported by computer networks $[2,4]$. Other important concepts for implementing Plug and Play Business are:

- Internet communities: Enterprises dynamically join a Plug and Play Business community by installing and running the Plug and Play Business software and by describing and validating the resources of the enterprise, e.g., production capacity, distribution network, intellectual capital, etc. The community is dynamic in the sense that enterprises may (in principle) join and leave the community at any time. However, there may be a need for a specific gate-keeper that enhances security by regulating the entering and leaving of the community, thus making it a semi-open artificial society [8].

- Roles: Each member of the Plug and Play Business community plays one or more roles, e.g., innovator, supplier/provider (of goods, services, expertise, etc.), distributor, marketer, financier, seller, etc. The choice of role depends on the 
company's core competencies and business intentions. An important role in the life cycle of businesses is the entrepreneur and we make a distinction between this role and that of the innovator. One of the main purposes of Plug and Play Business is to automate as much of the entrepreneurial role as possible, for instance by using intelligent agent technology, thus increasing the probability of turning an innovative idea into a business.

- Crystallization: A member of the Plug and Play Business community, typically an innovator, may at any time initiate an attempt to form a collaborative coalition inbetween the members (similar to the broker within VE breeding environments [3]). This process may be viewed analogous to crystallization, where a catalyst (innovator) initiates a process resulting in a precise form of collaboration, i.e., the formation of a VE. The main role of the entrepreneur, which to a large extent is automated by the Plug and Play Business software, is to drive this process. It may be a more or less elaborate process starting with just a seed of an innovative idea without any pre-defined business structure, or it may be a full-fledged business idea with well-defined needs to be met by potential collaborators.

Plug and Play Business has some resemblance to the work described by Chituc and Azevedo [7] in that dynamic collaboration processes for agile VEs are emphasized. However, this work excludes crucial aspects such as the formation of VEs and security management. The concept of Plug and Play Business is also similar to the concept of breeding environments as described by Camarinha-Matos and Afsarmanesh [2,3]. However, one distinction is that Plug and Play Business software focuses on promoting innovations by automating as much as possible of the entrepreneurial role in the VE formation and collaboration process. They state that "there is a need for flexible and generic infrastructures to support the full life cycle of VEs, namely the phases of creation, operation and dissolution" [3] and we believe that the Plug and Play Business software has the potential of constituting such an infrastructure. Moreover, they provide further motivation for our work by emphasizing the need for research on generic, interoperable, pervasive, free (low cost) and invisible (user-friendly) infrastructures that include methods for the creation of business (e.g., negotiation, methodologies for transforming existing organizations into a VE-ready format) and business collaboration (e.g., coordinated and dynamic resource sharing, administration and management of distributed activities and risk management).

\subsection{The Requirements of Plug and Play Business Software}

In order to realize the vision of Plug and Play Business, the software should enable the different community members to use their resources efficiently in VEs. We will now specify a set of functional requirements for the Plug and Play Business software. These requirements are extracted from interviews with representatives for SMEs, but are also based on previous work, e.g., on requirements for breeding environments (cf. [3]).

First of all, a Plug and Play Business community requires certain general functionalities. One example is the gate-keeper facility that regulates the entering (and leaving) of enterprises and registers them as members of the community. Moreover, a surveillance mechanism that monitors the behavior of members may be necessary in order to cope with malicious users. 
In the VE formation (crystallization) phase, when the catalyst initiates the VE, the following functions are helpful in forming a successful collaborative coalition:

- Finding: To find candidates suitable for a potential collaboration alliance is an important function. This function primarily concerns the catalyst of the business idea to provide the requirements of the preferred abilities of a potential collaborating partner. The finding functionality may include the possibility both for search, based on specific needs specified by criteria, e.g., role, type of products, and business model, as well as for posting general needs or ideas that other members may suggest solutions and/or resources for. Further, the software should also provide the feature of suggesting actors for collaboration based on, for example, content-based recommendation and collaborative recommendations.

- Evaluation: When a set of potential collaborators have been found they need to be evaluated. This requires support for using track records and potentially support for certification schemes of, for instance, the trustworthiness of the actors. Further, decision support for evaluating trade-offs between a number of characteristics are needed, e.g., trade-offs between cost of product/service, cost of transportation, and time to delivery of product/service. Which actors to choose for the formation of a VE should be based on the evaluation and the estimated value of collaboration with other actors in the alliance.

- Negotiation: When the catalyst has selected actors for the necessary roles of the $\mathrm{VE}$, agreements between the actors with respect to financial and products/service transfers need to be settled. The Plug and Play Business software should provide support for different types of contracts of agreement including support for intellectual property rights.

When the crystallization phase is finished and a VE is formed, the Plug and Play Business technology should provide support also for the collaboration phase, i.e., the management of the actual business activities within the VEs. This support may be on a quite shallow level, e.g., transactions of information between actors. On a deeper level, the Plug and Play Business software should support and facilitate complex coordination and synchronization of activities. A wide range of information types needs to be transferred in an efficient way in order to reduce the administrational costs of the actors as well as reducing the risk of inaccuracy in information. The management of the VE requires support for controlling the flow of activities between the involved actors. It concerns activities with potential long-term consequences (e.g., initiating product development) as well as regular business activities (e.g., decisions of production and distribution). With respect to enterprise collaboration, the Plug and Play Business software must support the following (functional) requirements:

- Information resource-sharing: This is related to the content and purpose of the exchanged information with tasks ranging from administrative information exchange to complex operations planning. An example of a simple administrative task is ordering and invoicing, whereas a more complex task may concern making critical information available to the cooperating partners in order to improve operations by better and more efficient planning and scheduling, i.e., resource optimization.

- Multi-lateral collaboration: The more parties involved in the cooperation, the more complex the solutions may be. The simplest case concerns cooperation between only two enterprises, whereas the general case involves a large number 
of enterprises cooperating with each other in different ways (many-to-many cooperation).

\subsection{Exploring the Quality Attributes of Plug and Play Business Software}

In addition to the functional requirements presented above, there are some relevant non-functional requirements that should be met which we will discuss in terms of quality attributes, i.e., software attributes that can be measured using some metric. Based on interviews with SMEs and on previous work by Camarinha-Matos and Afsarmanesh [3], we believe that the following quality attributes are important for Plug and Play Business software:

- Scalability. A Plug and Play Business community may be very large and the number of involved enterprises can be large.

- Flexibility. Being adaptable or variable is important due to the heterogeneity of enterprises, relationships and actors in a VE. Hence, Plug and Play Business software must be flexible to the varying needs of member organizations.

- Performance. Although there may not be many hard real-time requirements for the Plug and Play Business software to meet, response times and other delays must be kept on a reasonable level.

- Cost. High costs associated with joining and participating in a community is considered an obstacle for any growing network [16].

- Usability. A user-friendly interface is crucial in order to get interaction from the humans involved in the chain of collaboration.

- Confidentiality. Prevention of unauthorized disclosure of information is a crucial security aspect of the software (and of the prosperity of the community).

- Integrity. As with the latter attribute, prevention of unauthorized modification of information is a critical characteristic of the Plug and Play Business software.

- Availability. Prevention of unauthorized withholding of information indicates that all security-enhancing mechanisms must be carefully considered so that they do not interfere with the other quality attributes.

The choice of system architecture is closely related to the system's performance in terms of a number of these attributes. Compared to a centralized architecture, a distributed architecture supports many of the quality attributes, e.g., flexibility, scalability and dynamicity. Also, the risk of single point of failure may be avoided increasing the robustness of the system.

A decentralized paradigm such as peer-to-peer (P2P) may be preferable for the Plug and Play Business software, because no central authority determines how the participants interact or coordinates them in order to accomplish some task. A P2P infrastructure self-configures and nodes can coordinate autonomously in order to search for resources, find them and interact together. P2P being a paradigm that allows building dynamic overlay networks, it can be used in order to realize an environment that manages a dynamic network of business relations. Dealing with business sensitive assets (e.g., innovators' knowledge), searching and retrieval of contents, as well as discovery, composition and invocation of new services, should be made secure and trustable. The $P 2 P$ infrastructure realizes an environment in which every organization can make its knowledge and services available to other organizations keeping control over them. In a P2P infrastructure, each organization 
can autonomously manage this task without having to delegate it to an external central authority that could be perceived as less trusted than the organization itself, and should be the object of an external (to the collaborating network) agreement between all the involved organizations.

\section{A Formal Model of Plug and Play Business}

In order to make the concept of Plug and Play Business more precise (so that the requirements of Plug and Play Business software can be crisply defined), we will now formalize the most relevant aspects. A Plug and Play Business community, $p$, can be described as a tuple:

where

$$
p=\langle A, R, V E, g\rangle
$$

- $A=\left\{a_{i}, \ldots, a_{n}\right\}$ is the set of actors (typically enterprises) in the community,

- $R=\left\{r_{1}, \ldots, r_{m}\right\}$ is the set of roles that the actors can play,

- $V E=\{v e 1, \ldots, v e l\}$ is the set of virtual enterprises currently active in the community, and

- $g$ is the gatekeeper facility that regulates the entering (and leaving) of actors to (and from) the community. In order to become a member of $p$ there is a set of criteria that must be fulfilled, e.g., VAT number must be declared, and business intentions and information systems must be specified. Thus, some of the aims of the gatekeeper are to ensure that this type of information is available to the Plug and Play Business community and to verify the identity of the actors.

Note that all these entities change dynamically, but with different frequency. New virtual enterprises may be formed (and dissolved) relatively frequently, actors enter and leave the community every now and then, and new roles may be added although this is not expected to happen often.

Moreover, we can describe an actor as a triple:

$$
a=\langle h, i, b\rangle
$$

Where $h$ is the person representing the actor/enterprise, $i$ is the relevant information systems, and $b$ is the Plug and Play Business client software (possibly composed of a set of intelligent agents and supporting an (agent) communication language, a set of relevant interaction protocols/patterns, a set of relevant ontologies, etc.) acting on behalf of the actor/enterprise.

Finally, we can describe a virtual enterprise as a pair:

$$
\text { ve }=\left\langle A R_{v e}, O_{v e}\right\rangle
$$

where

- $A R_{v e}$ is a set of pairs $\left\langle a_{i}, r_{j}\right\rangle$ where $a_{i} \in A$ and $r_{j} \in R$ i.e., the actors and their roles in the virtual enterprise, and

- $O_{v e}=\left\{O_{1}, O_{2}, \ldots O_{n}\right\}$ is a set of obligations for each of the $n$ actors to the virtual enterprise.

In Figure 1, we illustrate an example of a Plug and Play Business community consisting of five actors of which three have formed a virtual enterprise. 


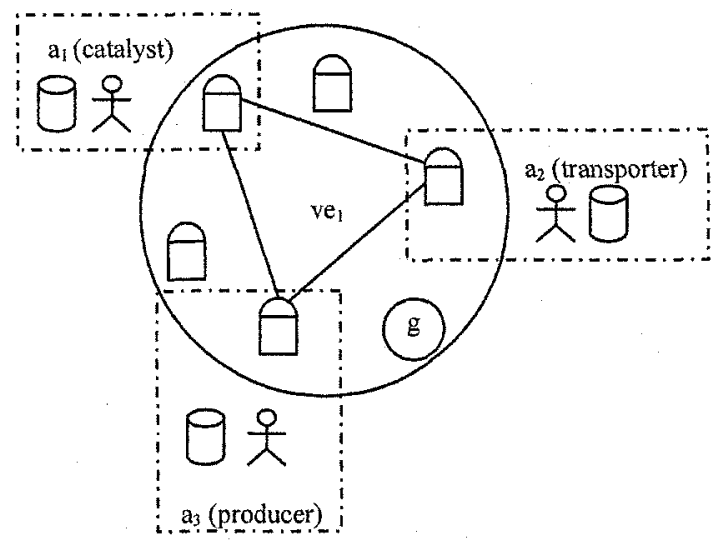

Fig. 1. An example of a Plug and Play Business community.

As mentioned earlier, there are two main activities that are supported by the Plug and Play Business software, namely (virtual enterprise) formation and collaboration. We will now formalize the different tasks involved in these activities using the model presented above.

\subsection{Formation}

Formation consists of three subtasks and is initiated by a catalyst, $c$, where $c \in A$.

- Finding requires that $c$ has a list of the roles that must be filled in order get a functional/working virtual enterprise. (This list is provided by $h_{c}$, i.e., the person representing $c$.) Then for each of the roles, the task for $b_{c}$ is to find the set of candidate actors $K$ where $K \subset A$ that are able to play the role.

- In the evaluation task, $c$ should rank the actors in $K$ according to a set of requirements $Q_{r}$ where $Q_{r}=\left\{q_{1}, q_{2}, \ldots q_{k}\right\}$ (provided by $p_{c}$ ). (Alternatively, $c$ may select the one with the highest rank $k$ where $k \in K$.)

- The goal of negotiation is to establish an agreement between $c$ and $k$ concerning $k$ 's set of obligations $O_{k}$ in $v e$.

\subsection{Collaboration}

We separate between two levels of collaboration: administrational and operational. They are defined by the type of interaction protocols they support. Administrational collaboration includes only protocols using the "weaker" performatives, such as, ask, tell, reply, etc. Let us call this set of interaction protocols $I P W$. Operational collaboration supports protocols also using the performatives that actually manipulate the receiver's knowledge, such as, insert, where the sender requests the 
receiver to add the content of the message to its knowledge base, and delete, where the sender requests the receiver to delete the content of the message from its knowledge base. Let us call this set of interaction protocols IPS. Moreover, we make a distinction between bi-lateral and multi-lateral collaboration. Thus, we have four types of collaboration:

- Bi-lateral administrational collaboration between two actors $a_{i}$ and $a_{j}$ (where $a_{i} \in A$ and $a_{j} \in A$ ) in a virtual enterprise ve should support the use of a set of interaction protocols, $I P W_{i j}$ where $I P W_{i j} \subset I P W$, between the two actors' information systems ( $i_{i}$ and $i_{j}$ ) and mediated by the actors' Plug and Play Business client software $\left(b_{i}\right.$ and $\left.b_{j}\right)$.

- Multi-lateral administrational collaboration between a set of actors $A_{u}$ (where $A_{u} \subset A$ in a virtual enterprise ve should support the use of a set of interaction protocols, IPW where $I P W_{u} \subset I P W$, between all the actors' information systems and mediated by the actors' Plug and Play Business client software.

- Bi-lateral operational collaboration between two actors $a_{i}$ and $a_{j}$ (where $a_{i} \in A$ and $a_{j} \in A$ ) in a virtual enterprise ve should support the use of a set of interaction protocols, $I P S_{i j}$ where $I P S_{i j} \subset I P S$, between the two actors' information systems ( $i_{i}$ and $i_{j}$ ) and mediated by the actors' Plug and Play Business client software ( $b_{i}$ and $b_{j}$ ).

- Multi-lateral operational collaboration between a set of actors $A_{u}$ (where $A_{u} \subset A$ in a virtual enterprise ve should support the use of a set of interaction protocols, IPS where IPS $\subset$ $\subset I P S$ between all the actors' information systems and mediated by the actors' Plug and Play Business client software.

\section{Useful Technologies}

Based on the functional requirements and the quality attributes, we hereby make a brief review of some relevant technologies that might be useful when developing Plug and Play Business software.

\subsection{Finding and evaluating potential partners}

The tasks of finding and evaluating (e.g., business partners) have been the object of a lot of research within the area of recommendation systems (cf. [1]). Here, the main idea is to automate the process of "word-of-mouth" by which people recommend products or services to one another. Recommendation systems are usually classified based on how they are constructed into three categories: content-based recommendation (based on previous interests of actors), collaborative recommendations (based on preference of similar actors), and hybrid recommendations (a combination of the two previous ones). So far, recommendation systems have successfully been deployed primarily in consumer markets (see, for instance the collaborative filtering system at book-dealer Amazon.com).. As most 
existing recommendation systems are not developed for $\mathrm{B} 2 \mathrm{~B}$ applications, they generally exclude the negotiation process. As recommendation systems are already deployed in large-scale consumer systems it can be assumed that they enable scalability, flexibility, usability and cost-efficiency. Thus, they may be a beneficial alternative to use when meeting the requirements of VE formation. Also, since they can take the history of a potential collaborator into account they may also contribute to the enhancement of security and trust.

In the area of intelligent agents, middle agents or brokering agents $[19,20]$ have been used to locate other agents in an open environment like the Internet. Here, each agent in the community typically advertises its capabilities to some broker. These brokering agents may simply be match-makers or yellow page agents who match advertisements to requests for advertised capabilities. Brokering agent systems are able to cope quickly and robustly with a rapidly fluctuating agent population [21], which indicates both a high level of flexibility, scalability, robustness and performance. This may make them appropriate to use in Plug and Play Business software.

\subsection{Establishing an agreement}

There is a long tradition in the area of agent-based systems of studying this topic, for instance using the Contract Net protocol [17] and computational auctions [15]. Auctions are generally considered to be a useful technique for allocating resources to agents [21]. However, they are too simple for many settings as they are mainly concerned with the allocation of goods or resources. For more general settings, where agents must reach agreements on matters of mutual interest and including complex constraints, richer techniques for reaching agreements are required. Here, negotiation may be a promising alternative (cf. [12] for an overview). Four different components are relevant for the Plug and Play Business setting:

- A negotiation set, which represents the space of possible obligations that agents can make.

- A protocol, which defines the legal obligations that the agents can make.

- A collection of strategies, one for each agent, which determines what obligations the enterprises will make.

- A rule that determines when the negotiation is over and the deal has been closed.

Here, the concept of obligations is an important component. Much work on obligations has been done, for instance in the area of electronic contracts, which are to be regarded as virtual representations of traditional contracts, i.e., "formalizations of the behavior of a group of agents that jointly agree on a specific business activity" [5]. Electronic contracts usually have a set of identified roles to be fulfilled by the parties involved in the relation. Three types of norms can exist within a contract structure, namely obligation, permission or prohibition. Plug and Play Business software focuses on obligations, i.e., that an agent has an obligation towards another agent to bring about a certain state of affairs before a certain deadline.

Agent-based auctions, negotiation protocols and electronic contracts may be sound technologies to enable the establishment of agreements within Plug and Play Business since intelligent agents can be designed to cope with individual goals and 
conflicting behavior (which may occur in the Plug and Play Business community). Also, they usually are fit for flexible and scalable interaction.

\subsection{Collaboration}

Several examinations on current state of the art technologies useful for building ICTinfrastructures with the purpose of business collaboration within VEs have been undertaken (cf. $[2,3,11]$ ). Some common conclusions are that multi agent technology constitutes a promising contributor to the development of support infrastructures and services. Internet and web technologies, such as web services, represent a fast growing sector with large potential in inter-enterprise collaboration support. However, further support in terms of supporting multi-lateral collaboration is necessary. A number of other emerging technologies, e.g., service-oriented architectures, the semantic web and countless collections of software standards (cf. the ebXML framework) are likely to provide important contributions. However, as stated by Camarinha-Matos and Afsarmanesh [2] "publicly funded research should avoid approaches that are too biased by existing technologies".

It seems that Microsoft's BizTalk Server [14] is the most sophisticated solution for inter-enterprise collaboration widely available. BizTalk is based upon a central server through which all exchanged information passes, it uses XML and supports the main protocols for email and http. However, BizTalk does not support multilateral communication and it is not fit for interoperable information resource sharing. Also, being a centralized proprietary client-server solution it has several disadvantages, such as, making the actors dependent of third party, being expensive and having possible risks for communication bottlenecks thereby failing to meet requirements such as scalability, flexibility, robustness, cost and security.

Based on our previous review [11], we believe that there are some technologies that may be useful for the collaboration task within Plug and Play Business software. One promising alternative for multi-lateral collaboration is the use of decentralized intelligent agents. As mentioned in the introduction, Davidsson et al. [10] describes a general wrapper agent solution based on open source freeware that makes it possible (in principle) for any business system to exchange (administrational) information with any other business system. Carlsson et al. [6] suggests further improvements to the wrapper agent technology by addressing security issues as well as an extended, possibly dynamic, set of involved companies and higher levels of cooperation (i.e., operational resource sharing). Another possibility is to use computational auctions $[15,0]$. They can be used within the collaboration task as a method for dynamically solving resource allocation within the VE. Possibly, auctions can also be deployed within multi-lateral administrational collaboration when allocating work tasks inbetween partners of a VE. Table 1 summarizes candidates candidate technologies for meeting the functional requirements of Plug and Play Business software. 


\begin{tabular}{|l|l|l|}
\hline \multicolumn{2}{|c|}{ VE Formation } & \multirow{2}{*}{ VE Collaboration } \\
\hline $\begin{array}{l}\text { Finding and evaluation } \\
\text { Recommendation systems }\end{array}$ & $\begin{array}{l}\text { Establishing agreement } \\
\text { Auctions negotiation } \\
\text { protocols and electronic } \\
\text { contracts }\end{array}$ & $\begin{array}{l}\text { Wrapper agents based on } \\
\text { service-oriented } \\
\text { architectures } \\
\text { (eb)XML }\end{array}$ \\
\hline
\end{tabular}

Table 1. Possible candidate technologies for addressing the functional requirements.

\section{Conclusions and Future Work}

One of the main weaknesses in the area of VEs is the lack of appropriate theoretic definitions, formal models and consistent modeling paradigms. The main contribution of this paper is a formal model of VE formation and collaboration and their associated tasks. In approaching a technology platform for Plug and Play Business, we have also made an assessment of useful technologies and related work. Based on this review, we can conclude that some of the evaluated technologies may be used for the tasks of Plug and Play Business software. With respect to finding and evaluating partners for a VE, recommendation systems show numerous fruitful examples that can be applied. For the process of establishing an agreement inbetween the catalyst and the highest ranked actor in the evaluation process, the Contract Net protocol and broker agents may be promising alternatives. Relevant approaches for supporting collaboration include Microsoft's BizTalk solution, wrapper agents and computational auctions.

The next step will mainly focus on further analyzing the components of the Plug and Play Business software and refine the non-functional requirements that were only briefly discussed. We will also continue to refine the formal framework presented above. In particular, we will further develop the concepts of roles and obligations in the context of Plug and Play Business. Also, what finally constitutes the technology platform of Plug and Play Business remains to be determined.

\section{Acknowledgments}

This work has been funded by the project "Integration of business information systems", financially supported by "Sparbanksstiftelsen Kronan". The authors would also like to thank all the members in the project.

\section{References}

1. G. Adomavicius and A.Tuzhilin, Toward the Next Generation of Recommender Systems: A Survey of the State-of-the-Art and Possible Extensions, IEEE Transactions on Knowledge and Data Engineering 17(6), $734-749$ (2005). 
2. L. M. Camarinha-Matos and H. Afsarmanesh, Collaborative Networks: A New Scientific Discipline, Journal of Intelligent Manufacturing 16, 439-452 (2005).

3. L. M. Camarinha-Matos and H. Afsarmanesh, Elements of a Base VE Infrastructure, Journal of Computers in Industry 51(2), 139-163 (2003).

4. L. M. Camarinha-Matos and H. Afsarmanesh, The Virtual Enterprise Concept. Infrastructure for Virtual Enterprises: Networking Industrial Enterprises (Kluwer Academic Publishers, London, 1999).

5. L. C. Cardoso and E. Oliveira, Virtual Enterprise Normative Framework Within Electronic Institutions, in: ESAW, Lecture Notes in Artificial Intelligence, Vol. 3451, edited by M.-P. Gleizes, A. Omicini, and F. Zambronelli (Springer Verlag, Berlin Heidelberg, 2005), pp. 14-32.

6. B. Carlsson, P. Davidsson, A. Jacobsson, S. J. Johansson and J. A. Persson, Security Aspects on Inter-Organizational Cooperation Using Wrapper Agents, in: Proceedings of the Workshop on Agent-Based Technologies and Applications for Enterprise Interoperability (ATOP) at the $4^{\text {th }}$ International Joint Conference on Autonomous Agents and Agent Systems (ACM Press, New York, 2005), pp. 13-25.

7. C.-M. Chituc and A. L. Azevedo, Enablers and Technologies Supporting SelfForming Networked Organizations, in: Interoperability of Enterprise Software and Applications - Workshops of the INTEROP-ESA International Conference EI2N, WSI, ISIDI and IEHENA 2005 edited by H. Panetto (Hermes Science Publishing, London, 2005), pp. 77-89.

8. P. Davidsson, Categories of Artificial Societies, Engineering Societies in the Agents World II, in: Lecture Notes in Computer Science, Vol. 2203, (Springer Verlag, Berlin, 2001), http://www.ide.bth.se/ ppdv/Papers/ESAWII.pdf.

9. P. Davidsson, A. Hederstierna, A. Jacobsson, J. A. Persson et al, The Concept and Technology of Plug and Play Business, in: $8^{\text {th }}$ International Conference on Enterprise Information Systems (forthcoming 2006).

10. P. Davidsson, L. Ramstedt, and J. Törnquist, Inter-Organization Interoperability in Transport Chains Using Adapters Based on Open Source Freeware, in: Interoperability of Enterprise Software and Applications, edited by D. Konstantas, J. -P. Bourrières, M. Léonard, and N. Boudjlida (Springer Verlag, Berlin, 2005), pp. $35-43$.

11. A. Jacobsson, Towards Integrated ICT-Support for Implementing Virtual Enterprises (Blekinge Institute of Technology, Ronneby, Sweden, Work in progress).

12. N. R. Jennings, P. Faratin, A. R. Lomuscio, S. Parsons, C. Sierra and M. Wooldridge, Automated Negotiation: Prospects, Methods and Challenges, International Journal of Group Decision and Negotiation 10(2), 199-215 (2001). 
13. H. Leibenstein, Entrepreneurship and Development, The American Economic Review 58, 72-83 (1968).

14.Microsoft BizTalk Server: http://www.microsoft.com/biztalk/ Last checked: 2006-02-27.

15. J. S. Rosenschein G. and Zlotkin, Rules of Encounter: Designing Conventions for Automated Negotiation among Computers (MIT Press, Cambridge MA, 1994).

16. C. Shapiro and H. R. Varian, Information Rules: A Strategic Guide to the Network Economy (HBS Press, Boston, MA, 1999).

17.R. G. Smith, The Contract Net Protocol: High-Level Communication and Control in a Distributed Problem Solver, IEEE Transactions on Computers C-29(12), 1104$1113(1980)$.

18.J. Tidd, J. Bessant, and K. Pavitt, Managing Innovation - Integrating Technological, Market and Organizational Change (John Wiley \& Sons, Chichester, England, 2005).

19.G. Wiederhold, Mediators in the Architecture of Future Information Systems, IEEE Transactions on Computers 25(3), 38-49 (1992).

20. H.-C. Wong and K. P. Sycara, A Taxonomy of Middle-Agents for the Internet, in: Proceedings of the Fourth International Conference on Multi-Agent Systems (IEEE, Piscataway, 2000), pp. 465-466, http:/citeseer.ist.psu.edu/cache/papers/cs/14301/http:zSzzSzwww.cs.cmu.eduzSzPeo plezSzsoftagentszSzpaperszSzMiddleAgentsICMAS2000.pdf/wong00taxonomy.pdf.

21.M. Wooldridge, An Introduction to MultiAgent Systems (John Wiley \& Sons, Chichester, England, 2004).

22. L. Yamamoto, Automated Negotiation for On-Demand Inter-Domain Performance Monitoring, in: Proceedings of $2^{\text {nd }}$ International Workshop on Inter-Domain Performance and Simulation (IPS 2004, Budapest, 2004), pp. 159-169, http://www.6qm.org/files/wp5/papers/ips2004.pdf. 